\title{
Récit(s) en fragments. Logiques sérielle et mondaine dans Little Nemo in Slumberland de Winsor McCay
}

\section{Alain Boillat}

\section{(2) OpenEdition}

Journals

Édition électronique

URL : http://journals.openedition.org/edl/576

DOI : 10.4000/edl.576

ISSN : 2296-5084

Éditeur

Université de Lausanne

\section{Édition imprimée}

Date de publication : 15 décembre 2013

Pagination : 93-124

ISBN : 978-2-940331-33-8

ISSN : 0014-2026

\section{Référence électronique}

Alain Boillat, «Récit(s) en fragments. Logiques sérielle et mondaine dans Little Nemo in Slumberland de Winsor McCay », Études de lettres [En ligne], 3-4 | 2013, mis en ligne le 15 décembre 2016, consulté le 20 décembre 2020. URL : http://journals.openedition.org/edl/576 ; DOI : https://doi.org/10.4000/edl. 576 


\section{RÉCIT(S) EN FRAGMENTS. \\ LOGIQUES SÉRIELLE ET MONDAINE DANS LITTLE NEMO IN SLUMBERLAND DE WINSOR McCAY}

Cette étude examine dans une perspective narratologique le fonctionnement de la sérialité dans les planches de la bande dessinée Little Nemo in Slumberland, qui sont tiraillées entre l'autonomisation de l'épisode et l'inscription dans un récit plus vaste. Les implications du rythme hebdomadaire de parution y sont envisagées du point de vue des niveaux de temporalité ainsi que des principes de segmentation en épisodes et de liage entre ceux-ci. Des seuils sont repérés dans cette série dont le traitement varie sur le plan de la narrativité et des relations instaurées entre le texte des récitatifs et les images. La diversité de ces régimes fait la richesse de cette œuvre, et montre comment McCay a su exploiter les possibilités de son moyen d'expression, notamment en privilégiant pour ses représentations oniriques et spectaculaires une logique mondaine qui, comme on l'explique ici, n'est pas sans parenté avec les univers des jeux vidéo.

La série Little Nemo in Slumberland de Winsor McCay, que nous proposons d'examiner ici dans une perspective narratologique, fut publiée de façon continue dans le New York Herald (et d'autres journaux associés) à un rythme hebdomadaire sous forme de planches dominicales en couleurs du 15 octobre 1905 au 23 juillet $1911^{1}$. La relative persistance de ce titre qui compte au total environ 450 planches et la légitimité qu'il a acquise ultérieurement en raison notamment de la virtuosité et

I. L'édition utilisée pour cette étude est la suivante: Little Nemo 1905-1914, Köln, Taschen Verlag, 2000. Précisons que la série de McCay se poursuivra au-delà des planches du New York Herald à un rythme de parution irrégulier jusqu'en 1914 sous le titre In the Land of Wonderful Dreams dans le New York American, puis refera provisoirement surface sous son titre original de 1924 à 1926 dans le New York Herald Tribune. 
de l'inventivité graphiques de $\mathrm{McCay}^{2}$ font de cette série l'un des plus importants comics de la période des pionniers de la première décennie du vingtième siècle. Dans la présente étude, nous nous intéresserons en particulier à la manière dont McCay y développe un récit en jouant avec la fragmentation imposée par le support de diffusion, et comment la narrativité s'installe à la fois à l'intérieur de chaque livraison et dans la durée à travers la publication successive d'épisodes qui, racontés en une planche, s'achèvent irrémédiablement sur une case de petit format nous montrant Nemo qui se réveille.

\section{La série: fragmentation et cohésion}

Chaque planche de Little Nemo raconte les aventures vécues en rêve par le personnage titre de la série. La ponctuation conclusive de chaque épisode s'incarne systématiquement dans l'image du petit garçon dans son lit, dont parfois il choit. Il s'agit là, littéralement, de la "chute» qui caractérise toute anecdote comique; sur le plan visuel, elle favorise la suggestion d'un mouvement qui raccorde fréquemment avec la fin de la dernière action du rêve (fig. 1), la discontinuité foncière du médium étant exploitée comme une saute entre les mondes, tandis qu'inversement la dynamique linéaire de la lecture lance une passerelle entre le rêve et la réalité qui repose sur la suggestion d'un mouvement continu. L'animation proposée le 15 octobre 2012 sur la page d'accueil du moteur de recherche Google à l'occasion du $107^{\mathrm{e}}$ anniversaire de la première parution de Little Nemo - célébration révélatrice de la popularité que connaît actuellement la série de McCay - mettait en exergue le parcours descendant du personnage (une nécessité liée au support de la page recréée sur Internet) qui, à la fin d'une péripétie, «retombe sur terre»: l'internaute était invité à cliquer sur le dessin d'une languette pour faire

2. Il est révélateur que Nemo constitue l'unique illustration de la couverture du catalogue de l'exposition organisée au Musée des Arts décoratifs de Paris en 1967 sous la direction de Claude Moliterni (P. Couperie, P. Destefanis, E. François, M. Horn, C. Moliterni, G. Gassiot-Talabot, Bande dessinée et figuration narrative). La «redécouverte" de McCay en France à la fin des années 1960 se concrétisera dans l'édition de Little Nemo chez Pierre Horay en 1969. Notons qu'une série récente, scénarisée par un historien de la bande dessinée, prend McCay pour héros en le projetant dans l'univers fictionnel de Little Nemo (cf. J.-Ph. Bramanti, Th. Smolderen, McCay). 
RÉCIT(S) EN FRAGMENTS

95

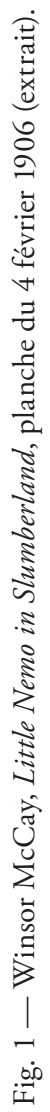


dérouler verticalement en plusieurs phases successives le paysage traversé par Nemo, puis perdait toute maîtrise sur le développement de l'action, le jeune héros étant précipité en chute libre dans l'océan, avant de poursuivre sa course vers l'abîme dans la dernière case où, après la saute de l'intercase, il était précipité hors de sa couche ${ }^{3}$. Comme l'a très justement souligné Thierry Groensteen, le lit en forme de "L» (première lettre du titre) étant constamment situé dans la partie gauche de l'ultime case et orienté en direction de l'extérieur de la planche (à l'instar du regard du garçon), la composition de la dernière vignette suggère graphiquement une ouverture sur l'épisode suivant ${ }^{4}$. La répétition inlassable du même procédé de clôture, emblématique d'un processus de standardisation propre aux séries de l'époque, s'accompagne par conséquent d'une volonté d'appeler la concaténation des épisodes.

Le personnage récurrent, Nemo, dont l'éternel retour dans la partie supérieure de la planche après avoir quitté le rêve dans la dernière case de l'épisode précédent assure une forme de continuité (qui n'est pas nécessairement narrative), contribue à individualiser la série et à fidéliser le lectorat. Car Nemo est, dans l'histoire de la bande dessinée, l'un des premiers héros individualisés de série. Or le marché de ce moyen d'expression appartenant à la culture de masse sera par la suite principalement régi, tant au niveau des parutions périodiques que des compilations en albums, par un principe sériel: qu'il s'agisse de Superman aux USA, de Lucky Luke dans le milieu de l'édition franco-belge ou de Tetsuwan Atom (Astro, le petit robot) au Japon, c'est sous la bannière d'un personnage éponyme que se fédèrent les très nombreux épisodes, parfois dus à des scénaristes et dessinateurs différents des auteurs originels. L’univers représenté - et en particulier le personnage titre, défini par

3. Little Nemo in Googleland, disponible sur http://www.google.com/logos/2012/ nemo/nemo12.html (consulté le 25 novembre 2012). Notons qu'en conjuguant la segmentation en strips et en cases avec une animation partielle de l'ensemble (qui n'est pas sans rappeler les «tableaux mécanisés» des spectacles de lanterne magique) ponctuée de phases d'immobilité, cette «séquence» morcelée proposée par Google signifie l'appartenance de cette représentation à des modèles de la fin du XIX ${ }^{\mathrm{e}}$ siècle tout en la faisant advenir sur un écran par des moyens informatiques. La vogue actuelle de "remédiation" d'un médium par un autre (au sens de J. D. Bolter, R. Grusin, Remediation) n'est ici pas sans suggérer un lien entre Little Nemo in Slumberland et les productions vidéoludiques contemporaines, comme nous le montrerons en fin d'article.

4. Th. Groensteen, «Nemo à nu», p. 53. 
un certain nombre d'attributs fixes - se fait le garant d'une cohérence indépendamment du style graphique, voire du médium utilisé 5 .

Winsor McCay privilégie indéniablement les composantes proprement visuelles de son moyen d'expression. A l'instar du réalisateur de films George Méliès à la même période ${ }^{6}$, il envisage la représentation fictionnelle comme le lieu du spectaculaire. La notion "d'attraction", associée par les historiens du cinéma aux premiers temps du médium ${ }^{7}$, s'applique tout autant aux planches de McCay qui s'inspire, pour concevoir l'univers onirique de Nemo, de l'apparence clinquante et factice des parcs d'attractions de Coney Island, ainsi que de la démesure des paysages urbains des métropoles américaines ${ }^{8}$. Le dessinateur exploite en effet de façon optimale la taille imposante de son support $(53,5$ centimètres sur $41,5)$ et l'excellente qualité d'impression des couleurs dont bénéficient les tirages de la grande presse. Dans le catalogue de la récente exposition consacrée à McCay au Cartoonmuseum de Bâle, Alexander Braun note à propos de l'adaptation en comédie musicale de Little Nemo prévue en 1908 pour la gigantesque salle de l'Hippodrome de New York (5000 places) que la bande dessinée s'y prêtait particulièrement dans la mesure où les promoteurs du show s'intéressaient avant tout «à un scénario qui offrait suffisamment d'occasions pour des scènes spectaculaires». Il poursuit ainsi :

5. Ce type de phénomènes est particulièrement perceptible de nos jours, à l'ère du développement horizontal des industries du divertissement, qui exploitent certaines franchises à travers plusieurs médias et produits dérivés.

6. Méliès déclarait par exemple rétrospectivement à propos de la tâche qui fut la sienne: "Celui qui compose un sujet cinématographique de fantaisie doit être un artiste, épris de son art, ne reculant devant aucun travail [...], cherchant avant tout à faire disparaître le scénario sous les arabesques dans lesquelles il l'enveloppe ainsi qu'un peintre fait disparaître la toile du tableau sous les touches artistiques de sa palette» (in L'Echo du cinéma, 7 [31 mai 1912]; nous soulignons).

7. Outre les travaux d'André Gaudreault, citons l'article fondateur de T. Gunning, "The Cinema of Attractions».

8. L'un et l'autre peuvent d'ailleurs être pensés conjointement: Rem Koolhaas a bien montré dans New York Délire que la construction de gratte-ciel s'inscrivait dans un imaginaire fantasmatique identique à celui qui anima les promoteurs des parcs de Coney Island. A travers le regard ludique qu'il porte sur la ville, McCay met selon nous en exergue cette filiation inavouée. 
Ils faisaient des affaires en bombardant de sensations optiques un public ébahi. C'était là exactement la nature du comics Little Nemo in Slumberland .

La dominante attractionnelle des planches de McCay, exacerbée par la vivacité des couleurs utilisées, n'exclut pas que les planches témoignent également, dans une certaine mesure, d'une inscription dans l'ordre du récit, ne serait-ce que pour offrir un cadre général à l'exhibition du merveilleux, un canevas permettant d'organiser la découverte de Slumberland. La notion de récit est souvent définie à l'aune de paramètres comme la cohérence, la causalité, la vectorisation linéaire et l'unité textuelle dont on pourrait penser a priori qu'ils font défaut à un contenu transmis de manière fragmentée sous la forme d'épisodes. Toutefois la série, qualifiée par Bruno Monfort lorsqu'il étudie le cas de Sherlock Holmes de "produit d'une rencontre entre le récit et son support de publication " ${ }^{10}$, "est comme dotée, à travers la répétition, d'une fonction supra-narrative, puisque, par-delà la discontinuité des récits, c'est elle qui doit assurer au lecteur qu'il existe au moins potentiellement un rapport entre les textes intelligible selon des modalités très proches de celle d'un récit, même schématique ${ }^{11}$. McCay exploite la fonction cohésive d'un récit minimaliste en créant une matrice adaptée aux déclinaisons paradigmatiques induites par la sérialité.

Le "rapport entre les textes" mentionné par Monfort tend à se renforcer lorsque nous sommes en présence de la structure du feuilleton qui, en dépit d'une discontinuité intrinsèque, produit une forme d'unité (le récit fût-il ouvert ou inachevé) ${ }^{12}$; nous verrons toutefois que la série Little Nemo exploite très peu les ressorts du feuilleton. Précisons que nous distinguons ici, pour éviter la confusion qui règne parfois dans la terminologie courante lorsqu'il est question, par exemple, de productions télévisuelles, d'une part le principe du retour du même propre à la série, d'autre part le morcellement en épisodes se référant les uns aux autres par des phénomènes d'appel, de reprise et de relance qui définit l'histoire

9. A. Braun, Winsor McCay 1869-1934, p. 158 (nous traduisons).

Io. B. Montfort, "Sherlock Holmes et le plaisir de la non-histoire», p. 49.

II. Ibid., p. 48.

I2. Voir F. Revaz, "Analyse (trans)textuelle d'un objet discursif complexe». 
à suivre du feuilleton ${ }^{13}$. A cet égard, il nous semble que Little Nemo est tiraillé entre la pratique de l'autonomisation de l'épisode et l'inscription de chaque livraison dans un récit plus vaste: McCay met d'abord en place la première, puis développe progressivement la seconde, sans doute pour garantir à la série une certaine perpétuation dans le temps. Mais de quelle temporalité parlons-nous? C'est là un premier point à préciser dans le cas d'une telle publication périodique.

\section{Les trois temps de Nemo}

Comme l'analyse du récit nécessite la prise en compte de la dimension temporelle, il faut souligner le fait que le mode de production et de réception des planches de Little Nemo induit trois niveaux distincts, et qu'il faut, par conséquent, en ajouter un à la distinction usuelle formalisée par Gérard Genette entre «temps de l'histoire» et «temps du récit" ${ }^{14}$. Il est d'autant plus important de distinguer ces trois types de temporalité qu'ils interagissent constamment chez McCay, qui se plaît parfois à en rabattre l'un sur l'autre. Il s'agit des niveaux suivants:

- Le temps diégétique ("temps de l'histoire»), soit celui auquel sont soumis les personnages dans l'histoire racontée. Dans Little Nemo, il ne vaut que pour un monde "enchâssé», le héros n'ayant pas de vie actualisée en dehors de ses rêves et de son brusque réveil - ainsi lorsque les parents expliquent le cauchemar de l'enfant en faisant référence à ce qu'il a mangé la veille ${ }^{15}$, cette référence à un temps passé ne vaut que par ce qu'elle produit dans le présent de la chute.

- Le temps du récit, relatif à la place accordée dans le support physique à un contenu narratif donné, compris en général entre 3 et 6 strips de 1 à 4 vignettes par planche. Sur une même page, l'unité narrative est l'action, qui peut être cantonnée à une vignette unique ou se déployer sur plusieurs, s'achever à la fin d'un strip ou déborder sur le suivant. La comparaison entre ces deux premiers temps permet les observations

13. Les deux termes ne sont par conséquent pas exclusifs: certaines séries feuilletonnent, tandis que d'autres non (ou guère).

I4. G. Genette, Figure III, p. 77 sq.

I5. On retrouve là un motif présent dans une autre série de McCay, Les Cauchemars de l'amateur de fondue au Chester. 
habituelles quant au tempo narratif, ainsi que des constats quant à l'ampleur variable des ellipses qui interviennent entre chaque case et entre les strips. En ce qui concerne le passage d'une planche à l'autre, McCay tend à faire correspondre la temporalité diégétique indiquée dans le texte au troisième niveau de temporalité décrit ci-dessous.

- Le temps réel de la parution de la planche, vécu par le lecteur dans son quotidien (il ne s'agit donc pas seulement d'un temps abstrait de la lecture), qui dans Little Nemo est également pris en compte au niveau diégétique, selon un processus de changement de niveau (la frontière entre l'extradiégétique et l'intradiégétique est abolie) qui ressortit de la métalepse au sens de Genette ${ }^{16}$. Le temps de l'histoire peut être certes déduit des actions entreprises (sur la base de l'expérience que le lecteur peut avoir des actions représentées), mais lorsque les personnages évoquent le temps qui s'est écoulé, leur cadre de référence est celui de la publication hebdomadaire. En effet, le monde onirique se situe hors du temps, les seuls repères existant pour les protagonistes de Slumberland correspondant aux scansions introduites par le mode de diffusion des images. Aussi le découpage du récit en épisodes n'est-il pas nié par une pseudo-continuité diégétique, mais exhibé à l'intérieur même de la diégèse. Lorsque, dans l'épisode du 14 juin 1908, le personnage de la jeune Marie s'adresse à Nemo pour lui dire: "Calme-toi, Nemo! La princesse va arriver, après tout, ça ne fait que huit semaines de retard", c'est aussi au lecteur qu'elle demande de prendre son mal en patience, et c'est au rythme de parution auquel elle fait référence puisqu'elle apparaît déjà aux côtés du héros pour attendre la princesse huit semaines plus tôt, dans la planche du 26 avril. D’ailleurs, le 24 mai 1908, elle précisait: « [...] ça fait quatre semaines qu'on attend!». La vie des personnages est donc scandée par des ellipses de sept jours séparant des moments relativement brefs, condamnés à être interrompus par la limite d'une planche unique - contrainte qui est convertie en facteurs diégétiques comme le réveil involontaire de Nemo effrayé, ou l'irruption de Flip qui l'enjoint à se réveiller. Dans la dernière case de la planche du 25 février 1906 dont on verra le rôle charnière qu'elle joue sur un plan narratif (fig. 2), Nemo s'assoit sur les marches d'un portail fermé, une pancarte indiquant qu'il n'ouvrira que le dimanche suivant, tandis que le page situé

I6. G. Genette, Métalepse. 
Fig. 2 - Winsor McCay, Little Nemo in Slumberland, planche du 25 février 1906. 
de l'autre côté de la grille prend congé du héros en lui disant qu'ils se reverront le dimanche suivant.

Dans ce monde, seuls les jours de parution des sunday pages existent. En projetant le temps vécu sur l'univers diégétique, les auteurs de comics comme McCay mettent en scène la rencontre entre la sphère du lecteur et le monde du texte, nous incitant à accorder une attention toute particulière à la relation instaurée par l'œuvre avec son destinataire, qui est d'autant plus importante en bande dessinée qu'il incombe au lecteur de remplir mentalement les vides correspondant aux intercases et au passage d'une page à l'autre. De telles références au temps réel exacerbent ce que Paul Ricoeur appelle la "complexité du rapport [...] au temps de la fiction par rapport au temps de l'expérience phénoménologique», «la nécessité de disjoindre le système des temps [du verbe] de l'expérience vive du temps» et, en même temps, l'«impossibilité de l'en séparer complètement $"{ }^{17}$. Non seulement l'unité de la semaine constitue pour les personnages l'unique repère, mais les moments marquants de l'année en termes de rituels sociaux tels que la Saint-Valentin, le $1^{\text {er }}$ avril, Noël, la Fête nationale, etc. affectent explicitement le contenu de l'histoire parue le jour concerné, McCay s'y pliant avec une systématicité devenue contrainte. Un rapport d'immédiateté - propre aux supports de presse - et de complicité est noué ainsi avec le lectorat de Little Nemo in Slumberland.

\section{La narrativité au niveau de la planche: Sammy et Nemo}

Comme nous l'avons montré ailleurs ${ }^{18}$, la série précédente de McCay, Little Sammy Sneeze, dont la durée de parution fut éphémère ${ }^{19}$ mais qu'il nous paraît intéressant de comparer à Little Nemo pour dégager certaines particularités de celui-ci, présente un contenu narratif particulièrement ténu qui pousse à son paroxysme la réitération caractéristique des productions sérielles et l'exacerbation de la dimension attractionnelle.

17. P. Ricoeur, Temps et récit, II, p. 117.

I8. A. Boillat, "Le récit minimal en bande dessinée».

19. Little Sammy Sneeze paraît dans le supplément dominical du New York Herald du 24 juillet 1904 au 9 décembre 1906. 
Chaque épisode expose ce qui "explose»: on assiste à la peinture haute en couleur(s) des effets dévastateurs provoqués par l'éternuement démesuré du personnage éponyme. Ce surgissement du chaos constitue également l'une des constantes de Little Nemo, dont les premiers épisodes s'achèvent fréquemment sur l'apparition inopinée du soleil qui réduit Slumberland à néant.

Ici et là, c'est la récurrence du protagoniste principal, un enfant, qui donne son titre à la série. On sait à quel point le motif des facéties de garnements est prégnant dans les comics de l'époque ${ }^{20}$, dans l'héritage des Bildergeschichten de Wilhelm Busch. Cependant, contrairement aux intentions espiègles ou sadiques de ce type de personnage, Sammy et Nemo demeurent fort passifs, cantonnés à une fonction de spectateur d'actions effectuées devant eux par d'autres (tel est systématiquement le cas de Sammy et ponctuellement celui de Nemo, notamment lorsqu'il observe les défilés organisés en son honneur à Slumberland): alors que l'unique action du premier - par ailleurs irrépressible, c'est-à-dire mécanique, indépendante de la volonté du protagoniste - se résume au verbe compris dans son patronyme («Sneeze»), l'action d'éclat de Nemo («personne» en latin) consiste le plus souvent dans le fait de se réveiller. Certes, la série évoluera vers un renforcement de l'intentionnalité de Nemo, figure plus active de voyageur, sans doute pour éviter l'impasse du minimalisme de Little Sammy Sneeze. Mais dès les premiers épisodes, une différence significative se fait jour: contrairement à Sammy qui n'interagit aucunement avec son environnement si ce n'est en le bouleversant par l'éternuement, Nemo entretient une relation de désir avec le pays des rêves qu'il veut visiter (même s'il fait lui-même obstacle à cette quête en se réveillant), et surtout avec la princesse qu'il tente inlassablement de rejoindre. A cet égard, la série redouble, dans le rapport de Nemo à Slumberland, la relation supposée qu'entretient le lecteur avec le monde de l'œuvre.

Si les épisodes qui nous intéressent ici témoignent d'un fort schématisme, ce dernier peut être néanmoins rapporté à la construction d'un récit dont la série Little Sammy Sneeze, même si elle n'est pas exempte de narrativité et suscite chez le lecteur des phénomènes

20. Voir P. Chemartin, N. Dulac, «La figure du garnement aux premiers temps du comic strip et de la cinématographie». 
de suspense ${ }^{21}$, ne fait pas montre à ce même degré. Il en va comme si McCay s'en était tenu dans Little Nemo à une application à la fois littérale et passablement désincarnée ${ }^{22}$ du schéma actanciel de Greimas - sans doute parce que Little Nemo se nourrit pour une large part des archétypes du conte merveilleux, un genre qui servit d'étude de cas à l'approche proppienne dont s'inspire Greimas ${ }^{23}$. Dans la case initiale et faîtière qui fait également office de bandeau de titre et d'ornement de la planche, le roi Morphée assume son rôle de destinateur, et commande pour sa fille esseulée (destinatrice) la venue de Nemo, sujet dont les déplacements visent à rejoindre la princesse (objet du désir); en dépit des divers adjuvants mis à sa disposition (protecteurs, guides, moyens de locomotion), Nemo échoue en raison de frayeurs qui le conduisent à se réveiller (opposant). Dès lors toutefois que Nemo aura franchi le portail de Slumberland - inscrivant explicitement la série dans une chronologie narrative qui sera confirmée plus loin par la rencontre, longtemps différée, avec la princesse -, McCay introduit un opposant désolidarisé du sujet: "Hélas, il est dit que c'est à Slumberland que Nemo rencontrera son premier ennemi, en la personne de Flip", nous informe le récitatif du 4 mars 1906. Ce protagoniste jaloux incitera continuellement Nemo à se réveiller, ou fera intervenir son oncle, "soldat de la garde de l'aube" capable de dissiper la nuit (figure à laquelle correspondra plus tard un autre personnage récurrent, le Dr. Pilule, appliqué quant à lui à neutraliser Flip). L'apparition nuisible de Flip permet de garantir la brièveté et la clôture de chaque épisode, "diégétisant" la contrainte éditoriale. Le récit mènera toutefois à des modifications de ce schéma initial, Flip se rangeant finalement du côté de Nemo, l'accompagnant dans ses périples. Les premiers épisodes empruntaient déjà, à travers la découverte de Slumberland, au merveilleux (notamment à travers les variations d'échelle) des Voyages de Gulliver de Swift (1735); la série se développera dans le sens du renforcement d'un principe d'organisation

2I. Comme nous l'avons montré dans l'article suivant à paraître: A. Boillat, F. Revaz, "Intrigue, Suspense, and Sequentiality in Comic Strips».

22. En effet, les protagonistes y sont bien plus des fonctions que des êtres pétris dans la chair de la fiction auxquels le lecteur serait invité à s'identifier. Comme l'a noté T. Groensteen, «lire Little Nemo ne suppose pas une participation affective aux événements représentés: la bande se déchiffre et s’apprécie comme pur spectacle» («Nemo à nu», p. 43).

23. A. J. Greimas, Sémantique structurale; V. Propp, Morphologie du conte. 
hérité des récits de voyage. A partir du 21 août 1910, Nemo et son équipe cesseront d'explorer le monde des rêves pour voyager sur terre à l'aide d'un dirigeable, les villes se succédant après que la première destination, New York, a été atteinte. Nemo révise sa géographie, et la série tend à endosser une fonction plus pédagogique à l'intention du jeune lectorat.

Il faut aussi considérer, outre la dimension narrative, la composante proprement visuelle, qui joue un rôle important dans la mise en exergue de l'action. Alors que Little Sammy Sneeze obéit invariablement (et peut-être jusqu'à la parodie) à la régularité du découpage en gaufrier et au modèle de la décomposition chronophotographique du mouvement, Little Nemo in Slumberland se caractérise par une inventivité débordante sur le plan de la forme et du format des cases, le monumentalisme de la représentation étant fréquemment souligné par des images de grande dimension qui se détachent de la planche. De toute évidence fort conscient des deux niveaux de lecture de la bande dessinée - le linéaire (succession des vignettes) et le tabulaire (vision globale de la page) - qu'identifieront les sémioticiens ${ }^{24}$, McCay exploite le motif du gigantisme pour permettre une phase immédiate de saisie de la planche qui s'attache aux déplacements de personnages nettement mis en évidence, isolés par l'œil d'un arrière-plan qui ne sera examiné en détail qu'ultérieurement. Ce procédé consistant à accentuer un élément de l'image (souvent un corps humain, mais parfois aussi un véhicule) ${ }^{25}$ dans plusieurs cases successives afin de renforcer la continuité au niveau graphique se met en place rapidement, dès la sixième semaine de la série, avec le personnage de Bozo, dont les dimensions (même courbé, son dos touche la partie supérieure d'une vignette) et la tenue voyante (orange striée de noir) provoquent un effet de détachement par rapport au fond plus pastel (fig. 3). Nemo, lui aussi, bénéficie à plusieurs reprises d'une taille hors du commun comparativement à son environnement, réduit dans cet imaginaire enfantin au statut de jouets. Ainsi, dans la planche du 22 septembre 1907, Nemo et son compagnon le "petit sauvage" essaient-ils de quitter une ville miniature pour regagner le château (fig. 4). Afin de se repérer, notre héros décide de gagner les hauteurs de la ville en escaladant les immeubles. Le strip

24. A la suite de P. Fresnault-Deruelle, «Du linéaire au tabulaire».

25. Voir la voiture rouge de la planche du 29 novembre 1908, qui, dessinée dans toutes les orientations possibles, occupe une très grande part de chacune des cases. 
Fig. 4 - Winsor McCay, Little Nemo in Slumberland, planche du 22 septembre 1907. 
central de la planche, composé de quatre cases verticales de grand format, est consacré aux étapes de cette ascension. Sur le fond composé de façades monochromes dépourvues de motifs, les corps des deux géants font saillie, et les différentes positions qu'ils occupent, soulignées par l'ombre projetée, sont très distinctement perceptibles, même pour un regard balayant rapidement la page. La force de cette bande est de concilier l'exhibition tabulaire avec l'inscription de chaque image dans une linéarité. En effet, les quatre instants prégnants choisis pour représenter cette action dessinent une diagonale ascendante, et les trois dernières vignettes suggèrent, grâce aux postures de Nemo et à l'alternance gauche-droite, un mouvement de balancier que l'on associe spontanément à un phénomène continu (qui serait interrompu de façon régulière, comme un métronome). Or, en comparant dans un second temps les positions du personnage avec le décor sur lequel sa silhouette se détache, on peut déduire, sur le plan de la temporalité diégétique, d'importantes ellipses, Nemo gravissant dans chaque case un immeuble différent. En fait, les variations de la position de l'observateur par rapport à la scène que McCay introduit sciemment de façon discrète élident une partie du déplacement de l'enfant: alors que le cadrage semble constant (notamment en raison de la saturation de l'image), nous progressons en fait dans chacune des cases d'un étage correspondant à un immeuble complet, si bien que l'impression est donnée d'un mouvement unique, alors que nous saisissons dans un second temps qu'il s'agit, comme dans les planches photographiques de Muybridge, de phases distinctes "prélevées» sur plusieurs mouvements ${ }^{26}$, mais sélectionnées et agencées de telle sorte à produire l'effet de rendu d'un mouvement unique continu. Le dynamisme qu'introduit un tel traitement du découpage de l'action inscrit au cœur de nombreuses planches une vectorisation du parcours qui ne s'arrête pas au bas de la page, ainsi que le suggère ici le regard hors-champ des deux personnages orienté dans le sens de la lecture,

26. A propos des recompositions du photographe américain, voir la comparaison que propose Marta Braun entre Marey et Muybridge dans Picturing Time. Même si l'influence des travaux de Muybridge sur les pionniers américains des comics a souvent été mise en évidence - notamment par Th. Smolderen dans Naissances de la bande dessinée (p. 103-112) -, il faut bien sûr distinguer le prélèvement effectif résultant de la captation photographique de l'imitation de celui-ci par le dessin. Un strip comme celui dont il est question ici n'en témoigne pas moins d'une maîtrise par McCay des implications liées aux notions d'instant et d'intervalle. 
repris dans l'ultime case: c'est aussi l'épisode suivant qui est appelé par la représentation de protagonistes en perpétuel déplacement.

\section{D'une planche à l'autre: seuils, continuité et liage}

Dans la série Little Nemo, la circularité structure les vingt premiers épisodes, dont la première case située sous le bandeau de titre nous montre systématiquement Nemo dans son lit, déjà gagné par le rêve (dans lequel le lecteur est immergé d'entrée de jeu). La boucle consiste à quitter le lit déjà présent dans le monde onirique pour y revenir dans le monde réel. Or ce principe d'un retour au point de départ est abandonné à partir de la première vignette de la planche du 4 mars 1906 (fig. 5) où nous retrouvons Nemo là où nous l'avions laissé (fig. 2), soit sur le seuil du portail de Slumberland ${ }^{27}$. La disposition des personnages dans le décor est quasi identique, de sorte que nous avons l'impression d'assister à la phase ultérieure d'un même mouvement (Nemo s'est retourné, tandis que son guide a levé les bras); certes, comme nous l'avons noté, l'ellipse correspond à une durée diégétique d'une semaine, mais la succession des deux dessins présente une forte continuité qui est rare dans cette série, même au sein d'une même planche. L'éternel retour prend fin: Little Nemo sera désormais placé sous le signe de la progression de son héros dans l'exploration du royaume de Morphée. Une fois passé le seuil de Slumberland, nous ne retrouvons plus Nemo dans sa chambre en début de planche; McCay thématise à travers la figuration d'une série de portes - numérotées, elles correspondent aux étapes que doit successivement franchir Nemo - un changement de régime symptomatique de la tension existant dans ce type de publications entre l'autonomisation de la planche destinée à être consommée de manière indépendante et l'inscription des daily panels individuels dans une chaîne narrative établie au niveau de la série.

27. La célèbre planche du 26 juillet 1908, où les pieds du lit se transforment en gigantesques pattes, fait exception en marquant provisoirement le retour à une case inaugurale montrant la chambre de Nemo. 
110

ÉTUDES DE LETTRES

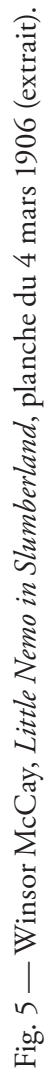


La reprise en début d'épisode du lieu sur lequel s'est achevé le précédent constitue un facteur décisif en termes de continuité ${ }^{28}$, de même que la récurrence d'un même moyen de locomotion: par exemple, le condor géant faisant office de monture qui apparaît à la fin de la planche du 22 avril 1906 - dont la composition scindée en deux parties par la ligne oblique de la rambarde de l'escalier suggère que la partie de droite est déjà celle d'un épisode à venir, comme s'il s'agissait d'un volet cinématographique entre deux séquences (fig. 6) - permettra de traverser quatre livraisons successives. Dans un univers fluctuant comme celui de Little Nemo in Slumberland, où les transformations subites qui affectent le monde lors du passage d'une case à l'autre sont aussi fréquentes que les "trucs" dans les films contemporains de Méliès (et que les métamorphoses créées par McCay dans ses premiers films d'animation, dès Little Nemo en 1911) ${ }^{29}$, certains éléments assurant la stabilité de la représentation jouent un rôle clé dans l'établissement d'une continuité. Dans l'épisode du 18 mars 1906, Flip, que l'on a déguisé en Nemo en lui faisant croire que ce travestissement l'aiderait à rejoindre la princesse, s'étonne, lorsqu'on le conduit dans la cour où l'attend une charrette rudimentaire tirée par deux oies, de ne pas disposer de l'équipement qui avait été préalablement mis à la disposition de celui dont il usurpe l'identité: "Où sont mes zèbres et mes paons? C'est ça mon attelage? Bon, j'espère que j'arriverai...». Cette réplique n'est pas sans rappeler au lecteur le somptueux attelage dont avait bénéficié Nemo la semaine précédente, l'effet comique résultant de l'opposition entre le

28. L'importance de cet effet de reprise dépend du degré d'invariance des caractéristiques de l'image, notamment au niveau du cadrage. Il arrive qu'exceptionnellement une case liminaire fasse office de rappel de l'épisode précédent: ainsi la première vignette de la planche du 25 juillet 1909, située au centre du bandeau de titre, reprendelle l'action de l'épisode précédent montrant les trois protagonistes fuyant un volcan qui entre en irruption. Sans doute ce retour est-il lié à la déception manifestée par Nemo le 18 juillet: «On rate un sacré spectacle!».

29. Notons que cette instabilité relève néanmoins de manifestations thaumaturgiques (ce n'est pas un hasard si Nemo sera affublé dans le film de 1911 d'une baguette magique). Par conséquent, elle est motivée au sein de la diégèse, contrairement à l'arbitraire systématique qui règne dans le comics Krazy Kat de Herriman (où les décors épurés changent brusquement à chaque case), qui débute dans la presse à la fin de la grande période de Little Nemo in Slumberland. Significativement, dans l'épisode du 27 octobre 1907 de Little Nemo, les modifications de l'environnement montagneux sont le fait de l'avancée du groupe. 
faste réservé à Nemo et les animaux offerts à Flip. L'étonnement manifesté par le protagoniste qui ne retrouve pas certaines composantes du monde présentées précédemment appartient aux «opérations de liage ${ }^{30}$ dont McCay émaille sa série.

On sait que les séries classiques de bandes dessinées franco-belges, qui parurent toutes originellement en feuilletons dans des périodiques tels que Spirou, Tintin ou Vaillant, recourent à des procédés de dynamisation de la lecture qui consistent en l'instauration de sutures en amont (rappel de l'épisode précédent dans un résumé liminaire, suggestion d'une étape antérieure du mouvement ou de l'action, relance à partir d'une donnée préalable...) et en aval (action laissée en suspens, interrogations formulées à propos de la suite des événements, pronostics...) ${ }^{31}$. Françoise Revaz discute dans un article récent différentes marques de liage propres au "récit suspendu» qui sont utilisées afin de "tisser une unité au-delà des fragments", "de confirmer somme toute que l'on est toujours dans la même histoire " ${ }^{32}$. Ce qui deviendra, dès les strips de l'âge d'or des comics dans les années 1930, une stratégie narrative et commerciale bien rôdée n'est que très peu à l'œuvre chez McCay, qui se contente d'insérer des renvois ponctuels aux épisodes précédents ${ }^{33}$, de reprendre à l'entame d'un épisode certains éléments de la planche précédente ${ }^{34}$ ou d'annoncer

30. Nous reprenons ici la terminologie de Jean-Michel Adam, développée dans une perspective d'analyse textuelle des discours (La Linguistique textuelle, chapitre 3). Le texte étant envisagé par Adam comme une unité cohérente, les critères qui satisfont selon lui à l'établissement d'une continuité (référentielle) sont plus restrictifs que ce que nous pouvons observer dans les épisodes d'une série, mais demeurent pertinents lorsqu'on les considère individuellement. A cet égard, une analyse de détail reste à faire sur notre corpus, ainsi que l'établissement d'équivalents visuels à différents types d'opération de liage par le langage verbal.

3I. C'est ce corpus et cette problématique que nous examinons dans le cadre de notre collaboration au projet de recherche «Le découpage de l'action. Analyse narratologique de périodiques de bandes dessinées (1946-1959) » dirigé par Françoise Revaz et soutenu par le FNS.

32. F. Revaz, «Le récit suspendu».

33. Le personnage de Flip dit par exemple au début de l'épisode du 13 janvier 1907, en référence à la dernière action de la semaine précédente: «M'asperger d'encre et filer comme ça, tu vas voir!».

34. Par exemple, Flip répète à peu de chose près dans la première case du 3 février 1907 ce qu'il a dit à la fin de l'épisode précédent. 
- toujours vaguement et sans emphase aucune - ce qui va suivre ${ }^{35}$. Braun souligne par exemple que le dessinateur ne recourt à aucun "cliffhanger " ${ }^{36}$ : jamais, en effet, la planche ne s'achève sur une interrogation à fonction de relance, ni sur l'interruption d'une action en cours de développement. Néanmoins, on note une évolution dans la manière d'opérer un liage entre les épisodes, en fonction de principes dont l'instauration est par ailleurs signifiée au niveau diégétique - on sait l'interpénétration constante dans l'œuvre de McCay du monde réel et des mondes fictionnels, chaque seuil franchi par le petit Nemo introduisant un mo(n)de spécifique, notamment au niveau de la place conférée au texte.

\section{Evolutions du dispositif verbo-iconique de Little Nemo}

Le franchissement du seuil diégétique figuré par le portail correspond à une nouvelle étape dans la gestion du dispositif verbo-iconique: le texte légendé qui accompagnait jusque-là chaque vignette et assurait une continuité entre les images ne résiste pas à l'intrusion de Nemo dans Slumberland. Si la numérotation des cases subsiste, le fléchage du parcours de lecture est amoindri par la disparition d'une segmentation du texte en énoncés incomplets qui, dans le mouvement de la lecture, appelaient le passage à l'extrait de texte suivant. Désormais, le texte attribué à une instance de narration extradiégétique est tout entier enclos dans le bandeau initial, qui se présente sous la forme d'un résumé programmatique de la planche dont la fonction devait notamment consister à assurer la lisibilité des actions. Par conséquent, soit le lecteur commence par ce texte et connaît d'avance l'histoire ensuite contée en images - même s'il nourrit quelque impatience à contempler dans le détail comment elle sera figurée, cette anticipation sape toute surprise -, soit il omet ce bloc de texte initial et se consacre à la lecture des cases dépourvues de légendes.

Parfois, l'amorce de ce texte contribue à intensifier les procédés de suture avec l'épisode qui précède. Ainsi la planche du 25 mars 1906

35. Ainsi la princesse déclare-t-elle, dans la dernière case du rêve de la planche du 2 décembre 1906, en observant la sorcière métamorphosée et Flip leurré par l'apparence de celle-ci : "J'ai idée qu'on va bien rire avec ces deux-là!».

36. A. Braun, Winsor McCay 1869-1934, p. 142. 
commence-t-elle par ces mots: «Après avoir fait face aux complications résultant de l'épisode du miroir, Nemo [...] file à toute allure...». La formule "épisode du miroir», réflexive en ce qu'elle souligne la nature fragmentée du récit ${ }^{37}$, fait office d'anaphore résomptive ${ }^{38}$ en renvoyant de façon synthétique au contenu de la planche précédente. Quant au processus inverse (la cataphore), il ne s'effectue dans ces textes inauguraux qu'en lien avec la planche elle-même, jamais avec l'un des épisodes ultérieurs (il faudrait pour cela que le texte puisse intervenir à la suite des images afin de ménager une relance).

Dans cette série où l'auteur ne craint pas d'introduire d'importantes modifications formelles, les textes du bandeau vont peu à peu changer de statut en se voyant plus intimement rattachés à l'iconique grâce à une diégétisation de leur profération: conversations téléphoniques, lectures de missives et autres communications d'émissaires permettent d'ancrer l'instance narrative dans le monde de la fiction, la voix narrative du récitatif étant dès lors prise en charge par un ou plusieurs personnages dans des phylactères (fig. 7) ${ }^{39}$. En dépit des temps verbaux au passé, les événements relatés n'ont pas encore eu lieu: le lecteur découvre peu après (à moins que son œil n'en ait déjà saisi les grandes lignes) la transposition visuelle de ces propos. En passant du bloc continu à une pluralité de bulles, le texte introductif se fait moins détaillé (il ne déflore plus complètement ce qui va suivre), et s'inscrit dans une interaction verbale où les encouragements de protagonistes optimistes quant à l'arrivée de Nemo tendent à instaurer une suture vers l'aval ${ }^{40}$. Dernière étape: le texte inaugural disparaît complètement le 29 juillet 1906 lorsque Nemo, enfin, aura rejoint la princesse et partira en balade avec elle. Le pronostic émis par la jeune femme au moment du baiser devait sans doute résonner pour le public adulte comme une promesse grivoise: «On ne va pas s'ennuyer tous les deux, mon doux Nemo!». L'ennui ne sera pas de la partie, en effet, tout comme le récitatif.

37. Parfois, la formule semble issue d'un feuilleton médiatique portant sur un fait divers, comme «l'affaire du policier somnolent de la semaine dernière» (22 avril 1906).

38. J.-M. Adam, La Linguistique textuelle, p. 105.

39. Bandeau de la planche du 29 avril 1907.

40. Par exemple dans la réplique suivante: «Nemo s'est réveillé à la dernière minute. Vous ne pourrez donc pas le voir avant la semaine prochaine» (17 juin 1906). 


\section{Logique sérielle, logique mondaine}

Dans Little Nemo, le moment où le héros est extrait du monde onirique est souvent figuré comme une destruction de ce monde. La série est sous-tendue par un paradoxe qui consiste à offrir un monde fastueux au lecteur pour le lui soustraire aussitôt. La baguette magique de McCay fonctionne dans les deux sens: dans un épisode, les serviteurs du roi construisent à partir de rien une cité imaginaire (23 mai 1909), alors que dans le suivant on "range la ville» comme on le ferait de jouets remisés dans un coffre (fig. 8-9). Dans un tel binôme, répété durant les deux semaines suivantes avec un autre décor, toute progression au cours de la série est annulée, alors que le retour au vide, à la quasi page blanche, s'impose. Si les cases de Little Nemo in Slumberland oscillent ainsi entre densification et raréfaction de leurs composantes, c'est que McCay ne cesse de rappeler à son lecteur qu'il s'agit d'un monde imaginaire, aussi factice que Coney Island, qu'un rien peut réduire à néant (d'où la fragilité de nombreux personnages dont on nous dit qu'ils sont faits de verre ou de sucre).

Au vu de l'importance conférée au franchissement de frontières à l'intérieur même du monde des rêves (lui-même distinct de la case du réveil) et de l'extrême variété des environnements traversés par Nemo, il nous semble que ce qui prime chez McCay est une logique "mondaine" (un univers de fiction conçu ici comme pluri-univers) ${ }^{41}$ qui assure la cohérence de la série tout en permettant les plus folles déclinaisons. Si la dimension narrative n'est pas si centrale, c'est parce que ce sont avant tout les multiples facettes de la diégèse qui fascinent. A cet égard, la série de McCay n'est pas sans partager certains traits avec les univers vidéoludiques d'aujourd'hui où, précisément, le récit (atténué par l'interactivité) fait place à la proposition de mondes dans lesquels le joueur est invité à s'immerger. Lancé comme un mobile à travers des décors mouvants, chutant d'une case à l'autre comme s'il descendait d'un étage, investi dans des actions réalisées en boucle, Nemo est un aïeul des avatars pixellisés qui sillonnent les actuels jeux de plateforme (comme le fameux Mario - présent lui aussi dans des productions en série -, il tente de

4I. Nous consacrerons prochainement un ouvrage à cette question dans le champ du cinéma: Cinéma, machine à monde. Essai sur les films à univers multiples (à paraître chez Georg, Genève). 
Fig. 8 - Winsor McCay, Little Nemo in Slumberland, planche du 23 mai 1909. 
Fig. 9 - Winsor McCay, Little Nemo in Slumberland, planche du 30 mai 1909. 
rejoindre celle qui l'attend à travers un parcours semé d'embûches). La mise en place d'une linéarité du trajet qui met fin à la répétition d'étapes similaires s'opère chez McCay lorsque son personnage atteint un seuil: une fois que Nemo a gagné les marches menant au portail, il repartira de là pour sa prochaine aventure. Ce procédé présente une parenté évidente avec le principe de sauvegarde (saved state) permettant aux usagers de jeux vidéo de stocker digitalement l'information afin que «lorsque le jeu est relancé plus tard, le joueur puisse continuer là où il s'était arrêté» ${ }^{42}$. Parmi les diverses modalités de fonctionnement de ce système de sauvegarde, on en trouve une qui consiste en un enregistrement intervenant automatiquement à certains niveaux atteints dans le(s) monde(s) du jeu; il s'agit là d'un principe très proche de celui qui sous-tend l'usage réflexif opéré par McCay à partir des différents seuils représentés concrètement par des portails. L'interaction entre le monde représenté par le média et celui du joueur est manifeste dans cette prise en compte du temps réel de l'usager; le lecteur de Little Nemo, lui, doit s'interrompre en fonction du critère prédéfini d'une semaine qui sépare la parution de chaque épisode. Comme le souligne Jesper Juul lorsqu'il distingue deux types de contraintes (relatives aux mondes de la fiction d'une part, au fonctionnement de l'interface vidéoludique d'autre part) ${ }^{43}$, le fait que Mario possède trois vies ne relève pas d'une donnée touchant à la diégèse, mais d'une règle du jeu; dans le cas des épisodes de comics, la clôture en fin de planche et l'ellipse d'une semaine reposent somme toute sur un principe analogue. Juul souligne le fait que ces deux niveaux peuvent être contradictoires, provoquant ainsi une forme d'incohérence; comme on l'a vu, McCay s'efforce par contre d'harmoniser via la métalepse les déterminations diégétiques (fictionnelles) et extradiégétiques (éditoriales).

Pour poursuivre cette comparaison, on pourrait dire que Little Nemo in Slumberland commence comme un jeu d'arcades purement attractionnel ${ }^{44}$, puis se développe dans la durée, par fragments, comme une succession de parties (même si le vertige visuel construit en crescendo

42. Http://www.wordiq.com/definition/Save_state (dernière consultation: 28 novembre 2012; nous traduisons).

43. J. Juul, Half-Real, p.130.

44. Comme l'explique M. Triclot: «L'arcade est le produit [d'un] déplacement: amener le jeu vidéo de l'université [les départements d'informatique] au parc d'attractions» (Philosophie des jeux vidéo, p. 127). 
demeure présent à l'intérieur de chacun des épisodes). On retrouve là, dans la succession d'une même série, deux régimes parents des modes de consommation vidéoludique. Cependant, la logique sérielle nous prémunit de tout game over: pendant plusieurs années, Nemo s'est remis en route chaque dimanche. Sa mort symbolique aux yeux des habitants du royaume de Morphée est, tout comme dans les jeux vidéo, oblitérée par les potentialités du multiple. La logique sérielle induit bien une activité de création et de lecture «ludique».

Alain BoIllat

Université de Lausanne 


\section{BIBLIOGRAPHIE}

Adam, Jean-Michel, La Linguistique textuelle, Paris, Armand Colin, 2011.

Boillat, Alain, "Le récit minimal en bande dessinée: l'histoire constamment réitérée d'un éternuement dans la série Little Sammy Sneeze de Winsor McCay", in Le Récit minimal. Du minime au minimalisme: littérature, arts, médias, éd. par Sabrinelle Bedrane, Françoise Revaz, Michel Viegnes, Paris, Presses Sorbonne Nouvelle, 2012, p. 103-117.

—, Cinéma, machine à monde. Essai sur les films à univers multiples (à paraître chez Georg, Genève).

Boillat, Alain, Revaz, Françoise, "Intrigue, Suspense, and Sequentiality in Comic Strips: Reading Little Sammy Sneeze» in Narrative Sequence in Contemporary Narratologies, ed. by Raphaël Baroni, Françoise Revaz, Columbus, Ohio State University Press (à paraître).

Bolter Jay David, Grusin Richard, Remediation. Understanding Media, Cambridge, MIT Press, 2000.

Bramanti, Jean-Philippe, Smolderen, Thierry, McCay, Paris, Delcourt, 2000-2006.

Braun, Alexander, Winsor McCay 1869-1934. Comics, Filme, Träume, Bonn, Exlibris, 2012.

Braun, Martha, Picturing Time: The Work of Etienne-Jules Marey (18301904), Chicago/London, University of Chicago Press, 1992.

Chemartin Pierre, Dulac Nicolas, "La figure du garnement aux premiers temps du comic strip et de la cinématographie», in Les Cases à l'écran. Bande dessinée et cinéma en dialogue, éd. par Alain Boillat, Genève, Georg, 2010, p. 125-148.

Couperie, Pierre, Destefanis, Protot, François, Edouard., Horn, Maurice., Moliterni, Claude, Gassiot-Tадвot, Gérard, Bande dessinée et figuration narrative: histoire, esthétique, production et sociologie de la bande dessinée mondiale, procédés narratifs et 
structure de l'image dans la peinture contemporaine, Paris, Musée des Arts décoratifs/Palais du Louvre, 1967.

Fresnault-Deruelle, Pierre, "Du linéaire au tabulaire", Communications, 24 (1976), p. 7-23.

Genette, Gérard, Figure III, Paris, Seuil, 1972.

—, Métalepse. De la figure à la fiction, Paris, Seuil, 2004.

Greimas, Algirdas Julien, Sémantique structurale, Paris, PUF, 1966.

Goensteen, Thierry, "Nemo à nu", in Little Nemo au pays de Winsor McCay, Toulouse, Editions du Milan, 1990, p. 42-53.

Gunning, Tom, «The Cinema of Attractions. Early Film, Its Spectator and the Avant-Garde», in Wide Angle, vol. 8, 3/4 (1986) (trad. française dans 1895, 50 (décembre 2006), p. 55-66).

Juul, Jesper, Half-Real. Video Games between Real Rules and Fictional Worlds, Cambridge, MIT Press, 2005.

Koolhans, Rem, New York Délire, Marseille, Editions Parenthèses, 2002 (1978).

McCaY, Winsor, Little Nemo 1905-1914, Köln, Taschen Verlag, 2000.

Montfort, Bruno, "Sherlock Holmes et le plaisir de la non-histoire", Poétique, 101 (février 1995), p. 47-67.

Propp, Vladimir, Morphologie du conte, Seuil, Paris, 1970 (1928).

Revaz, Françoise, "Analyse (trans)textuelle d'un objet discursif complexe: le feuilleton journalistique", in Congrès mondial de linguistique française, éd. par Jacques Durand et al., Paris, EDP Sciences, 2008, p. 1417-1428.

—, "Le récit suspendu: un genre narratif transmédial», in Genres et textes: déterminations, évolutions, confrontations, éd. par Michèle Monte, Gilles Philippe, Lyon, PUL (à paraître en 2013).

Ricoeve, Paul, Temps et récit, vol. II, Paris, Seuil, 1984.

Smolderen, Thierry, Naissances de la bande dessinée, Bruxelles, Impressions nouvelles, 2009.

Triclot, Mathieu, Philosophie des jeux vidéo, Paris, La Découverte, 2011.

Crédits iconographiques:

Fig. 1-9:

(C) Taschen Verlag. 
